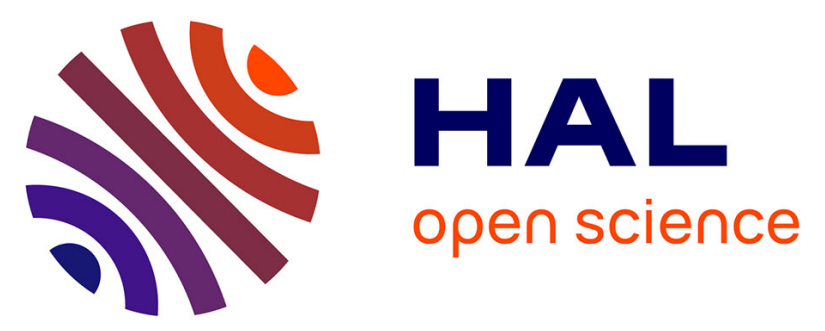

\title{
Investigation of the Tesla Transformer as a Device for One-Wire Power and Signaling and as a Device for Power and Signaling Through the Ground
}

Kaloyan Mihaylov, Rui Neves-Medeiros, Rumen Arnaudov, Stanimir Valtchev

\section{- To cite this version:}

Kaloyan Mihaylov, Rui Neves-Medeiros, Rumen Arnaudov, Stanimir Valtchev. Investigation of the Tesla Transformer as a Device for One-Wire Power and Signaling and as a Device for Power and Signaling Through the Ground. 6th Doctoral Conference on Computing, Electrical and Industrial Systems (DoCEIS), Apr 2015, Costa de Caparica, Portugal. pp.459-466, 10.1007/978-3-319-167664_49. hal-01343517

\author{
HAL Id: hal-01343517 \\ https://hal.inria.fr/hal-01343517
}

Submitted on 8 Jul 2016

HAL is a multi-disciplinary open access archive for the deposit and dissemination of scientific research documents, whether they are published or not. The documents may come from teaching and research institutions in France or abroad, or from public or private research centers.
L'archive ouverte pluridisciplinaire HAL, est destinée au dépôt et à la diffusion de documents scientifiques de niveau recherche, publiés ou non, émanant des établissements d'enseignement et de recherche français ou étrangers, des laboratoires publics ou privés.

\section{(ㄷ)(i)}

Distributed under a Creative Commons Attribution| 4.0 International License 


\title{
Investigation of the Tesla Transformer as a Device for One-Wire Power and Signaling and as a Device for Power and Signaling through the Ground
}

\author{
Kaloyan Mihaylov ${ }^{1}$, Rumen Arnaudov ${ }^{1}$, Stanimir Valtchev $^{2}$ \\ ${ }^{1}$ Department of Telecommunications, Technical University of Sofia, Bulgaria \\ ${ }^{2}$ Department of Electrical Engineering, UNINOVA, Nova University of Lisbon, \\ Caparica, Portugal \\ kaloyan.mihaylov@gmail.com, ra@tu-sofia.bg, ssv@fct.unl.pt
}

\begin{abstract}
A new method for power and communications through single wire or the ground as a medium is investigated. The focus of the research is based on the proper construction and operation of a Tesla Transformer, so as to be able to transmit power and/or signals through one wire or the ground. The conclusions are based upon experimental data. There are successful results for single wire power supply with efficiency of about $20 \%$. There is room for improvement of the circuits, so better efficiency is expected. The utilization of the soil as a medium for currents propagation didn't give any useful results. Due to its complex properties, the ground transmission requires lower working frequency, hence bigger and more complicated coils, special drive circuit and good grounding.
\end{abstract}

Keywords: single wire power, single wire signalling, power through the ground, ground communications, Tesla coil, Tesla transformer, quarter wave resonator.

\section{Introduction}

The idea for wireless electricity is not new. The first experiments in this field began in the end of the 19th century. The most fascinating proposal was the Tesla's idea to send electrical power to every point on the globe by utilizing a giant low-frequency transmitter [1]. Most of his experiments are associated with the production of very high voltages for fun, although this is not the case. As it will be seen below, the electrical discharge or sparking is an unwanted side effect of his famous transformer. However, the scale of the 
Tesla's construction doesn't permit easy reproduction of those effects. Scaled-down models could be used to investigate the working principle of the transformer.

The main topics presented in the paper are the transformers construction, single wire experiments and ground experiments. Chapters 3, 4 and 5 describe the basic principles that we have obeyed during the building of the coils and conducting the experiments, and in chapters 6 and 7 there are the results.

In chapter 3 it is stressed that the secondary coil of the transformer behaves like a distributed-elements circuit, not as lumped elements one.

In chapter 4 is represented the method to find the resonant frequency of the coil. It is the starting point for energy transmission, because other frequencies doesn't work.

Due to its nature, in chapter 5 we have suggested the most useful methods to calculate the secondary coil of a Tesla transformer. The fully theoretical approach isn't precise enough, therefore we have focused our attention on more robust methods.

In chapter 6 we have shown the results of single wire power transfer. There are successful results.

Chapter 7 is dedicated to ground transmission experiments. We haven't got any measurable result for ground transmission. Because there are some successful attempts for ground transmission by other researches, some directions were given.

\section{Relationship to Cloud-based Solutions}

The focus of cloud computing is usually computational performance, storage, network connectivity and collaboration. In order to deeply influence everyday life, a lot of small devices are brought in the network. Remote data acquisition and control techniques require a good network connectivity and power supply. However, there is no "one for all" solution. A lot of different tools exist for data or power transmission, like low-power wireless transceivers, optical transceivers and energy harvesting devices of different kind.

The aim of our experiments is to discover new methods for data transmission together with secure power supply. The single wire solution has shown its potentialities. Small portions of power were successfully transmitted over short distances. The true utilization of only one wire could enable further development and introduction of sensor nodes or electronic devices of other choice.

This method could be used in places where energy harvesting modules cannot bring the power needed to operate a device. Another advantage is the more robust communication between the devices (for example in noisy wireless environment).

Drawback of the system is the need of a conductor. However there are some developments that investigate the possibilities to use the ground instead of single wire. The circuits require much higher power levels and the propagation of the power is different from the single wire system, but the resonator coil and the principles are the 
same. Despite the lower frequencies, the ground is another medium that could be added to the existing communication solutions.

The results in this area of research give us conviction, that these methods will soon be ready to take place in the civil and industrial development, hence ensuring wider connectivity of different devices to the cloud.

\section{Common Construction Mistakes}

As it was stated above, the most common mistake is the consideration of the Tesla's resonator as a lumped element system [4]. Solution of the inductance and the capacitance for a lumped element would not reveal any useful information for the constructor. The most common formulas of Wheeler and Nagaoka are valid for DC or low-frequency designs.

In fig. 1 there is a typical example of poorly designed secondary. It consists of 5550 turns of $0.18 \mathrm{~mm}$ solid copper wire. Height of the coil is $1 \mathrm{~m}$, and the diameter of the coil is $2 \mathrm{~cm}$. The use of very thin wire leads to higher pure resistance, hence lower Q factor of the coil.

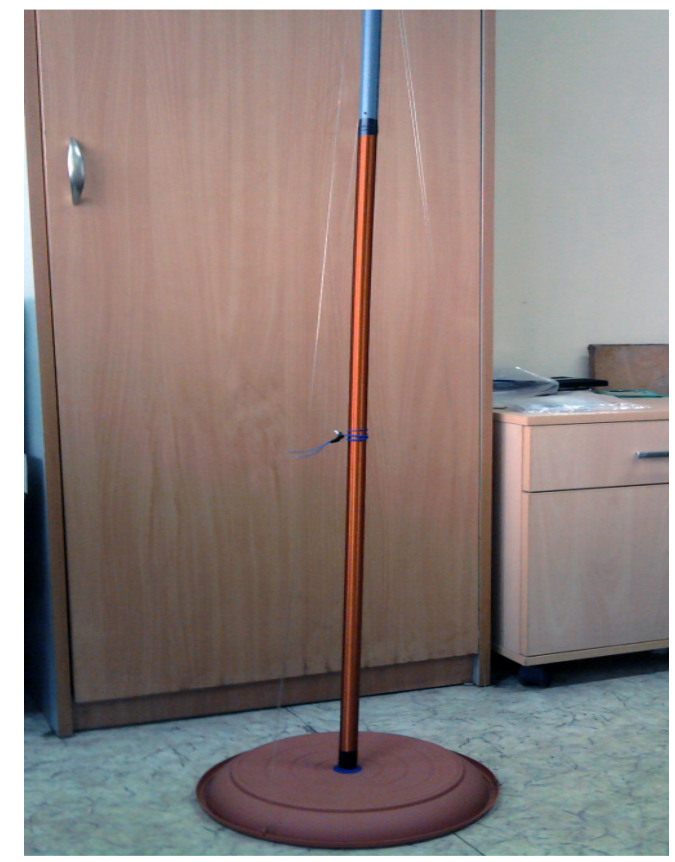

Fig. 1. Example of poorly constructed quarter-wave resonator coil. 
Another problem of such a coil is its height to diameter ratio. The higher length to diameter ratio increases the self-capacitance of the coil [6.3, p. 452]. It was found that the coil shown in fig. 1 has great amount of variable capacitance, dependent on the objects near the test setup. Therefore it has also low Q factor and weakly detected resonant frequency. According to [3, p. 452, table 1] the single-layer solenoids have lowest capacitance when their height to diameter ratio is about $0.5 \div 2$. Due to its high selfcapacitance, the coil could be used as a sensor.

\section{Experimental Approach for Detecting the Coil's Resonant Frequency}

Since the secondary of a typical Tesla Transformer is described by the distributed element approach, it should be indicated what current/voltage distribution is expected. In fig. 2 there is the general case of a typical secondary coil.
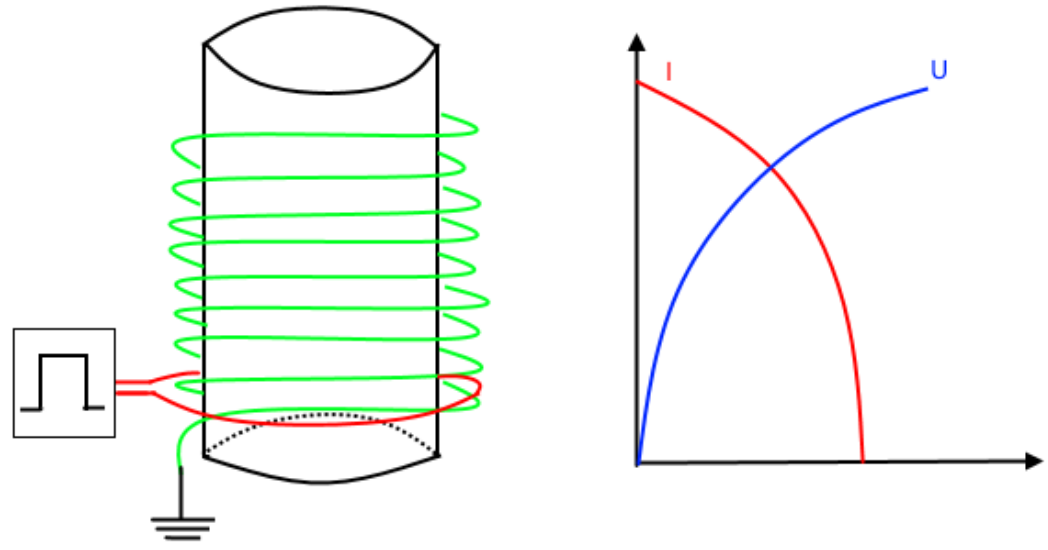

Fig. 2. Representation of the voltage-current distribution along a resonator coil.

The current in the base of the coil is high and diminishes along the height of the coil. The voltage in the base is small, but increases along the coil height.

If the primary coil is impulse-fed, then the secondary oscillates on its natural resonance. The output voltage is measured with a scope's probe on top of the coil, without direct connection. The grounding pinch of the probe should be removed for accuracy. Ordinary signal generator could be used, even simple PWM circuit as SG3525 or TL494 to drive the primary, but only to find the resonant frequency of the secondary. 


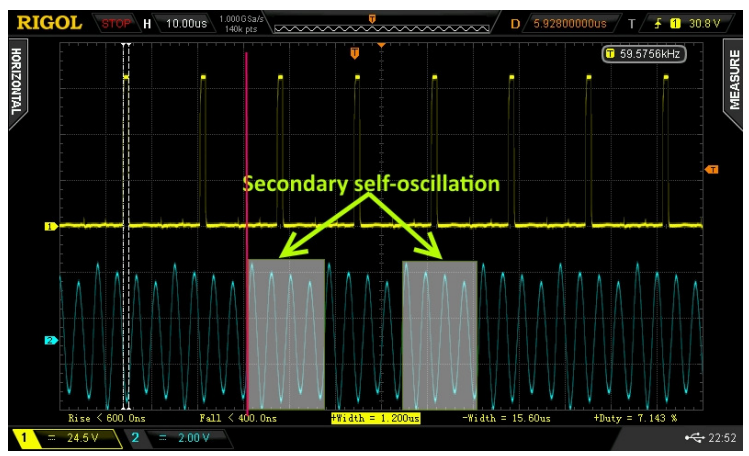

Fig. 3. Oscillogram of one of the driving channels of SG3525 and the response of the resonator.

In fig. 3 the oscilloscope probe \#1 is connected to one of the SG3525 outputs. The drive frequency is about $60 \mathrm{kHz}$. The second output is connected to a 1-turn primary of a arbitrary chosen air-core coil. Note: the phase difference between channel 1 and channel 2 is because of the second output used to monitor the drive frequency. The actual voltage waveform of the first output (connected to the primary) does not correspond to the waveform of channel 1, due to the loading. The drive impulse exact time is marked with a red line on the oscillogram.

The resonant frequency of the secondary coil is about $250 \mathrm{kHz}$. The experimenter should tune the drive frequency and duty cycle to achieve maximum amplitude. Also one must be careful of nearby objects around the coil. The human body strongly influences the parameters of the secondary, especially if it has a lot of turns and bigger self-capacitance.

\section{Calculation of the Secondary Self-Resonant Frequency}

In section 3 the most critical mistakes were given. To summarize, they are the use of thin wire and the high height to diameter ratio. Solid copper is preferred. The authors of the paper have the view that multistranded wire could be more suitable for the purpose if the separate strands are not insulated from each other. The insulation of the strands leads to higher inter-strands capacitance, hence lower frequency and Q of the coil.

The best method for coil calculation that we found suitable for our purposes is the GeoTC JavaScript library by the Tesla Secondary Simulation Project [2]. We have found that the calculations of the non-loaded bare (without top-load) secondary are within the pointed error limit. Other programs like E-Tesla6 (by Terry Fritz) could be more suitable if better accuracy is sought. A mixed Java/JavaScript web application based on GeoTC is FanTC (http://www.classictesla.com/fantc/fantc.html) which is used in our experiments. 


\section{Experimental Results for One-Wire Transmission}

To conduct the experiments two pieces of plane PCB transformers were used.

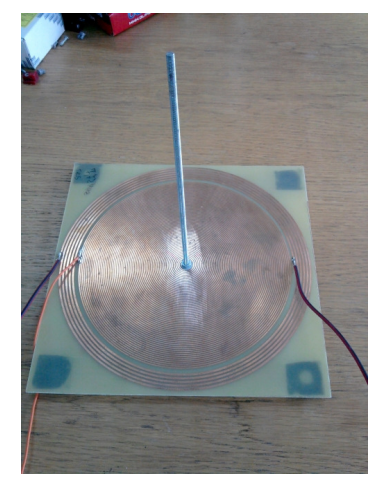

Fig. 4. Example of one of the transformers used in the experiments.

The characteristics of the two types of transformers are given in table 1 . In each of the cases the shorter coil (primary) is the exiting or load coil, and the longer coil (the secondary) is the resonator coil. The outer ends of the two secondaries are connected via $70 \mathrm{~cm}$ multistranded wire. The inner end of each secondary is connected to a $20 \mathrm{~cm}$ bolt used for capacitive loading of the resonator.

Table 1. Characteristics of the transformers.

\begin{tabular}{|l|l|l|}
\hline Parameter & Transformer 1 & Transformer 2 \\
\hline Number of turns (secondary) & 60 & 100 \\
\hline Wire width & $0.882 \mathrm{~mm}$ & $0.353 \mathrm{~mm}$ \\
\hline Turn spacing & $0.482 \mathrm{~mm}$ & $0.635 \mathrm{~mm}$ \\
\hline Inner diameter & 0 & 0 \\
\hline Outer diameter & $163.7 \mathrm{~mm}$ & $197.6 \mathrm{~mm}$ \\
\hline Length of wire & $15.426 \mathrm{~m}$ & $31.038 \mathrm{~m}$ \\
\hline DC Inductance & $193.3 \mu \mathrm{H}$ & $648.3 \mu \mathrm{H}$ \\
\hline
\end{tabular}

The circuit schematic is shown in fig. 5. R1 has the value of $10 \mathrm{Ohms}$ and is used to measure the input current. R2 has variable resistance and it is used to measure the maximum power point. The diode bridge is compound of 4 BAT754 Schottky diodes with $200 \mathrm{mV}$ of voltage drop for each diode. 


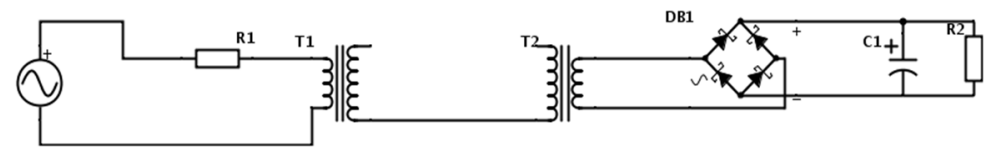

Fig. 6. Schematic of the connection of the transmitter and the receiver.

Part of the data for a test setup which utilizes Transformer 1 is shown in Table 2.

Table 2. Input and output power.

\begin{tabular}{|c|c|c|c|c|}
\hline Frequency & Input power & Output power & Efficiency & Note \\
\hline $5.19 \mathrm{E} 6$ & $11.5 \mathrm{~mW}$ & $0.87 \mathrm{~mW}$ & $8 \%$ & Loading - bare bolt \\
\hline $3.87 \mathrm{E} 6$ & $12.16 \mathrm{~mW}$ & $1.54 \mathrm{~mW}$ & $13 \%$ & $\begin{array}{c}\text { Loading - aluminium } \\
\text { can }\end{array}$ \\
\hline
\end{tabular}

In the "Note" column there is a remark of how the secondary coil is loaded. In order to achieve maximum $\mathrm{Q}$ of the coil, minimum capacitance should be introduced. However, it was found that some capacitance should be added for better energy transmission. The details of how exactly the capacitive loading influences the power transmission are not deeply studied yet.

Also it should be noted, that in the power balance there is a big amount of loss in the $\mathrm{R} 1$ resistor, which is used to measure the current in the primary coil and great voltage drop at the receiver's diode bridge. In table 3 the values of the experiments with "Transformer 2" styled coils are given.

Table 3. Values of experiments with Transformer 2.

\begin{tabular}{|c|c|c|c|c|}
\hline Frequency & Input power & Output power & Efficiency & Note \\
\hline $2.58 \mathrm{E} 6$ & $10.6 \mathrm{~mW}$ & $1.3 \mathrm{~mW}$ & $12.3 \%$ & Loading - bare \\
\hline $1.96 \mathrm{E} 6$ & $11.7 \mathrm{~mW}$ & $1.05 \mathrm{~mW}$ & $8.95 \%$ & Loading - can \\
\hline $1.93 \mathrm{E} 6$ & $8.09 \mathrm{~mW}$ & $1.8 \mathrm{~mW}$ & $22 \%$ & Loading - can \\
\hline
\end{tabular}

\section{Experimental Results for Ground Transmission}

$30 \mathrm{~cm}$ long, $8 \mathrm{~mm}$ diameter copper pipes were used for grounding the transmitter and the receiver coils. The test setup is shown in fig. 7.

There was no measurable output power. The diode bridge DB1 was removed so as the secondary of $\mathrm{T} 2$ to be open circuit. Then a measurement on both ends of the circuit was conducted with digital oscilloscope, but no signal was detected. 

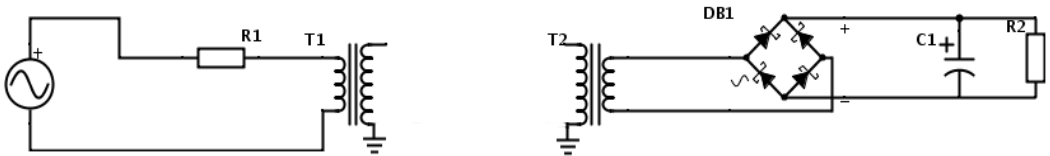

Fig. 7. Test setup of the ground transmission system.

At a distance of up to $3 \mathrm{~m}$ there was mutual inductance between the coils. Any presence of power cords pushes an error in the measurements. The human body and movements around the transformers does influence the working conditions of the system.

\section{Conclusions}

The objectives of the experiments were examination of the possibilities to use a quarterwave transformer as a power source for single wire power cord or power transmission through the ground. Two types of plane PCB transformers were used. To minimize RF interference and EM radiation from the system, the lower frequencies were preferred. Power levels of about $1 \mathrm{~mW}$ at the receiver's side were obtained, which satisfies some of the modern low-power electronic devices (such as the STM32L-series MCUs). These power levels are DC values after a bridge rectifier, so there is room for improvements.

The utilization of the system with ground as a medium for power transmission didn't gave measurable results. The measurements included bare oscilloscope connection to the receiver's secondary without amplifier. However, there are promising results of utilization of a $136 \mathrm{kHz}$ Tesla Coil transmitter with detectable signal at $5.5 \mathrm{~km}$ distance. [5]. Due to the properties of the soil, a different type of resonators should be used. The working frequency should be lower, hence bigger coils. The drive circuit should be optimized for higher power levels.

It should be noted that the aim of the experiments isn't maximum efficiency, but prove of the effect. Hence the system's performance is highly influenced by a lot of factors. The coupling between the primary and the secondary, mutual inductance, rectifier's efficiency, matching and other important factors are left out of consideration.

\section{References}

1. Tesla, N.: Patent \#787412: Art of transmitting electrical energy through the natural mediums (1905)

2. Nicholson, P. Tesla Secondary Simulation Project: Document pn2511.

3. Langford-Smith: Radiotron Designer's Handbook, p. 452. (1957)

4. Corum, K. L., Corum, J. F.: Tesla Coils and the Failure of Lumped-Element Circuit Theory (1999).

5. Peterson, G. L.: Yahoo! Group “wireless_energy_transmission”, post \#2083 